University of New Mexico

UNM Digital Repository

Mathematics and Statistics Faculty and Staff

Publications

Academic Department Resources

$11-2016$

\title{
Computation of Shortest Path Problem in a Network with SV- Trapezoidal Neutrosophic Numbers
}

\author{
Florentin Smarandache \\ University of New Mexico, smarand@unm.edu \\ Said Broumi \\ Assia Bakali \\ Mohamed Talea \\ Luige Vladareanu
}

Follow this and additional works at: https://digitalrepository.unm.edu/math_fsp

Part of the Logic and Foundations Commons, OS and Networks Commons, Other Computer Sciences Commons, Other Mathematics Commons, and the Set Theory Commons

\section{Recommended Citation}

Said Broumi, Assia Bakali, Mohamed Talea, Florentin Smarandache, \& Luige Vladareanu. (2016). Computation of Shortest Path Problem in a Network with SV-Trapezoidal Neutrosophic Numbers. In Proceedings of the 2016 International Conference on Advanced Mechatronic Systems, Melbourne, Australia, November 30 - December 3, 2016. 6 pages. http://doi.org/10.5281/zenodo.888855

This Article is brought to you for free and open access by the Academic Department Resources at UNM Digital Repository. It has been accepted for inclusion in Mathematics and Statistics Faculty and Staff Publications by an authorized administrator of UNM Digital Repository. For more information, please contact amywinter@unm.edu, Isloane@salud.unm.edu,sarahrk@unm.edu. 


\title{
Computation of Shortest Path Problem in a Network with SV-Trapezoidal Neutrosophic Numbers
}

\author{
Said Broumi \\ Laboratory of Information processing, Faculty of Science \\ Ben M'Sik, University Hassan II, B.P 7955, Sidi Othman, \\ Casablanca, Morocco \\ broumisaid78@gmail.com \\ Mohamed Talea \\ Laboratory of Information processing, Faculty of Science \\ Ben M’Sik, University Hassan II, B.P 7955, Sidi Othman, \\ Casablanca, Morocco \\ taleamohamed@yahoo.fr
}

\author{
Assia Bakali \\ Ecole Royale Navale, Boulevard Sour Jdid, B.P 16303 \\ Casablanca, Morocco. \\ assiabakali@yahoo.fr \\ Florentin Smarandache \\ Department of Mathematics, University of New Mexico, \\ 705 Gurley Avenue, Gallup, NM 87301, USA \\ fsmarandache@gmail.com; smarand@unm.edu
}

\author{
Luige Vladareanu \\ Institute of Solid Mechanics, Romanian Academy, Bucharest, Romania. \\ luigiv2007@yahoo.com.sg, luigiv@imsar.bu.edu.ro
}

\begin{abstract}
In this work, a neutrosophic network method is proposed for finding the shortest path length with single valued trapezoidal neutrosophic number. The proposed algorithm gives the shortest path length using score function from source node to destination node. Here the weights of the edges are considered to be single valued trapezoidal neutrosophic number. Finally, a numerical example is used to illustrate the efficiency of the proposed approach
\end{abstract}

Keywords- Single valued trapezoidal neutrosophic number; Score function; Network; Shortest path problem.

\section{INTRODUCTION}

In 1998, the concept of the neutrosophic set (NS for short) and neutrosophic logic were introduced by Smarandache in $[1,2]$ in order to efficiently handle the indeterminate and inconsistent information in real world. Neutrosophic set is a generalization of the theory of fuzzy set [3], intuitionistic fuzzy sets [4], interval-valued fuzzy sets [5] and intervalvalued intuitionistic fuzzy sets [6]. The concept of the neutrosophic set is characterized by a truth-membership degree $(\mathrm{t})$, an indeterminacy-membership degree (i) and a falsity-membership degree (f) independently, which are within the real standard or nonstandard unit interval $]^{-} 0,1^{+}[$. However, the neutrosophic theory is difficult to be directly applied in real scientific and engineering areas. To easily use it in science and engineering areas, Wang et al. [7] proposed the concept of SVNS, which is an instance of a neutrosophic set, whose functions of truth, indeterminacy and falsity lie in $[0,1]$. Recent research works on neutrosophic set theory and its applications in various fields are progressing rapidly [8].
Recently, based on the neutrosophic set theory, Subas [9] presented the concept of triangular and trapezoidal neutrosophic numbers and applied to multiple-attribute decision making problems. Then Biswas et al [10] presented a special case of trapezoidal neutrosophic numbers and applied to multiple-attribute decision making problems by introducing the cosine similarity measure. Deli and Subas [11] presented the single valued trapezoidal neutrosophic numbers (SVNnumbers) as a generalization of the intuitionistic trapezoidal fuzzy numbers and proposed a methodology for solving multiple-attribute decision making problems with SVNnumbers. In addition, Thamaraiselvi and Santhi [12] introduced a mathematical representation of a transportation problems in neutrosophic environment based on single valued trapezoidal neutrosophic numbers and also provided the solution method.

The shortest path problem (SPP) is one of the most fundamental and well-known combinatorial problems that appear in various fields of science and engineering, e.g, road networks application, transportation, routing in communication channels and scheduling problems. The main objective of the shortest path problem is to find a path with minimum length between any pair of vertices. The edge (arc) length of the network may represent the real life quantities such as, time, cost, etc. In a classical shortest path problem, the distances of the edge between different nodes of a network are assumed to be certain. Numerous algorithms have been developed with the weights on edges on network being fuzzy numbers, intuitionistic fuzzy numbers, vague numbers [13$16]$. 
Recently, Broumi et al. [17-22] presented the concept of neutrosophic graphs, interval valued neutrosophic graphs and bipolar single valued neutrosophic graphs. To this day, only a few papers dealing with shortest path problem in neutrosophic environment. The paper proposed by Broumi et al. [23] is one of the first on this subject. The authors proposed an algorithm for solving neutrosophic shortest path problem based on score function. The same authors [24] proposed another algorithm for solving shortest path problem in a bipolar neutrosophic environment. Also, in [25] they proposed the shortest path algorithm in a network with its edge lengths as interval valued neutrosophic numbers.

The goal of this work is to propose an approach for solving shortest path problem in a network where edge weights are charectreized by a single valued trapezoidal neutrosophic numbers .

In order to do, the paper is organized as follows: In Section 2, we review some basic concepts about neutrosophic sets, single valued neutrosophic sets and single valued trapezoidal neutrosophic sets. In Section 3, we propose some modified operations of single valued trapezoidal neutrosophic numbers. In section 4, a network terminology is presented, In section 5, we propose an algorithm for finding the shortest path and shortest distance in single valued trapezoidal neutrosophic graph. In section 6, we illustrate a practical example which is solved by the proposed algorithm. Finally, some concluding remarks are presented in section 7.

\section{PRELIMINARIES}

In this section, some basic concepts and definitions on neutrosophic sets, single valued neutrosophic sets and single valued trapezoidal neutrosophic sets are reviewed from the literature.

Definition 2.1 [1]. Let $X$ be a space of points (objects) with generic elements in $\mathrm{X}$ denoted by $\mathrm{x}$; then the neutrosophic set $\mathrm{A}$ (NS A) is an object having the form $\mathrm{A}=\left\{<\mathrm{x}: T_{A}(x)\right.$, $\left.I_{A}(x), F_{A}(x)>, \mathrm{x} \in \mathrm{X}\right\}$, where the functions $\mathrm{T}, \mathrm{I}, \mathrm{F}$ : $\mathrm{X} \rightarrow]^{-} 0,1^{+}$[define respectively the truth-membership function, an indeterminacy-membership function, and a falsitymembership function of the element $\mathrm{x} \in \mathrm{X}$ to the set $\mathrm{A}$ with the condition:

$$
{ }^{-} 0 \leq T_{A}(x)+I_{A}(x)+F_{A}(x) \leq 3^{+} .
$$

The functions $T_{A}(x), I_{A}(x)$ and $F_{A}(x)$ are real standard or nonstandard subsets of $]^{-} 0,1^{+}[$.

Since it is difficult to apply NSs to practical problems, Wang et al. [7] introduced the concept of a SVNS, which is an instance of a NS and can be used in real scientific and engineering applications.

Definition 2.2 [7]. Let $X$ be a space of points (objects) with generic elements in $\mathrm{X}$ denoted by $\mathrm{X}$. A single valued neutrosophic set A (SVNS A) is characterized by truthmembership function $T_{A}(x)$, an indeterminacy-membership function $I_{A}(x)$, and a falsity-membership function $F_{A}(x)$. For each point $\mathrm{x}$ in $\mathrm{X} T_{A}(x), I_{A}(x), F_{A}(x) \in[0,1]$. A SVNS A can be written as

$$
\mathrm{A}=\left\{<\mathrm{x}: T_{A}(x), I_{A}(x), F_{A}(x)>, \mathrm{x} \in \mathrm{X}\right\}
$$

Definition 2.3 [11]. A single valued trapezoidal neutrosophic number (SVTN-number) $\tilde{a}=<\left(\mathrm{a}_{1}, b_{1}, c_{1}, d_{1}\right) ; T_{a}, \mathrm{I}_{a}, \mathrm{~F}_{a}>$ is a special neutrosophic set on the real number set $R$, whose truth membership, indeterminacy-membership, and a falsitymembership are given as follows

$$
\begin{aligned}
& T_{a}(x)= \begin{cases}\left(x-a_{1}\right) T_{a} /\left(b_{1}-a_{1}\right) & \left(a_{1} \leq x \leq b_{1}\right) \\
T_{a} & \left(b_{1} \leq x \leq c_{1}\right) \\
\left(d_{1}-x\right) T_{a} /\left(d_{1}-c_{1}\right) & \left(c_{1} \leq x \leq d_{1}\right) \\
0 & \text { otherwise }\end{cases} \\
& I_{a}(x)=\left\{\begin{array}{lc}
\left(b_{1}-x+I_{a}\left(x-a_{1}\right)\right) /\left(b_{1}-a_{1}\right) & \left(a_{1} \leq x \leq b_{1}\right) \\
I_{a} & \left(b_{1} \leq x \leq c_{1}\right) \\
\left(\mathrm{x}-c_{1}+I_{a}\left(d_{1}-x\right)\right) /\left(d_{1}-c_{1}\right) & \left(\mathrm{c}_{1} \leq x \leq d_{1}\right) \\
1 \quad \text { otherwise } &
\end{array}\right. \\
& F_{a}(x)=\left\{\begin{array}{lc}
\left(b_{1}-x+F_{a}\left(x-a_{1}\right)\right) /\left(b_{1}-a_{1}\right) & \left(a_{1} \leq x \leq b_{1}\right) \\
F_{a} & \left(b_{1} \leq x \leq c_{1}\right) \\
\left(\mathrm{x}-c_{1}+F_{a}\left(d_{1}-x\right)\right) /\left(d_{1}-c_{1}\right) & \left(c_{1} \leq x \leq d_{1}\right) \\
1 \quad \text { otherwise } &
\end{array}\right.
\end{aligned}
$$

Where $0 \leq T_{a} \leq 1 ; 0 \leq I_{a} \leq 1 ; 0 \leq F_{a} \leq 1$ and $0 \leq T_{a}+I_{a}+F_{a} \leq 3 ; \mathrm{a}_{1}, b_{1}, c_{1}, d_{1} \in R$

Definition 2.3 [11]. Let $\tilde{A}_{1}=<\left(\mathrm{a}_{1}, a_{2}, a_{3}, a_{4}\right) ; T_{1}, \mathrm{I}_{1}, \mathrm{~F}_{1}>$ and $\tilde{A}_{2}=<\left(b_{1}, b_{2}, b_{3}, b_{4}\right) ; T_{2}, \mathrm{I}_{2}, \mathrm{~F}_{2}>$ be two single valued trapezoidal neutrosophic numbers. Then, the operations for SVTNnumbers are defined as below;

(i)

$\tilde{A}_{1} \oplus \tilde{A}_{2}=<\left(\mathrm{a}_{1}+b_{1}, \mathrm{a}_{2}+b_{2}, \mathrm{a}_{3}+b_{3}, \mathrm{a}_{4}+b_{4}\right) ; \min \left(T_{1}, T_{2}\right), \max \left(\mathrm{I}_{1}, \mathrm{I}_{2}\right), \max \left(\mathrm{F}_{1}, \mathrm{~F}_{2}\right)>$

(ii)

$\left.\tilde{A}_{1} \otimes \tilde{A}_{2}=<\left(\mathrm{a}_{1} b_{1}, \mathrm{a}_{2} b_{2}, \mathrm{a}_{3} b_{3}, \mathrm{a}_{4} b_{4}\right) ; \min \left(T_{1}, T_{2}\right), \max \left(\mathrm{I}_{1}, \mathrm{I}_{2}\right), \max \left(\mathrm{F}_{1}, \mathrm{~F}_{2}\right)\right)$

(iii)

$\lambda \tilde{A}_{1}=<\left(\lambda \mathrm{a}_{1}, \lambda \mathrm{a}_{2}, \lambda \mathrm{a}_{3}, \lambda \mathrm{a}_{4}\right) ; \min \left(T_{1}, T_{2}\right), \max \left(\mathrm{I}_{1}, \mathrm{I}_{2}\right), \max \left(\mathrm{F}_{1}, \mathrm{~F}_{2}\right)>$

A convenient method for comparing of single valued trapezoidal neutrosophic number is by use of score function.

Definition 2.4 [11]. Let $\tilde{A}_{1}=<\left(\mathrm{a}_{1}, a_{2}, a_{3}, a_{4}\right) ; T_{1}, \mathrm{I}_{1}, \mathrm{~F}_{1}>$ be a single valued trapezoidal neutrosophic number. Then, the score function $s\left(\tilde{A}_{1}\right)$ and accuracy function $a\left(\tilde{A}_{1}\right)$ of a SVTNnumbers are defined as follows: 
(i) $s\left(\tilde{A}_{1}\right)=\left(\frac{1}{12}\right)\left[a_{1}+a_{2}+a_{3}+a_{4}\right] \times\left[2+T_{1}-I_{1}-F_{1}\right]$

(ii) $a\left(\tilde{A}_{1}\right)=\left(\frac{1}{12}\right)\left[a_{1}+a_{2}+a_{3}+a_{4}\right] \times\left[2+T_{1}-I_{1}+F_{1}\right]$

Definition 2.5 [11]. Let $\tilde{A}_{1}$ and $\tilde{A}_{2}$ be two SVTN-numbers the ranking of $\tilde{A}_{1}$ and $\tilde{A}_{2}$ by score function is defined as follows:

(i) If $s\left(\tilde{A}_{1}\right) \prec s\left(\tilde{A}_{2}\right)$ then $\quad \tilde{A}_{1} \prec \tilde{A}_{2}$

(ii) If $s\left(\tilde{A}_{1}\right)=s\left(\tilde{A}_{2}\right)$ and if
(1) $\mathrm{a}\left(\tilde{A}_{1}\right) \prec a\left(\tilde{A}_{2}\right)$ then $\tilde{A}_{1} \prec \tilde{A}_{2}$
(2) $\mathrm{a}\left(\tilde{A}_{1}\right) \succ a\left(\tilde{A}_{2}\right)$ then $\tilde{A}_{1} \succ \tilde{A}_{2}$
(3) $\mathrm{a}\left(\tilde{A}_{1}\right)=a\left(\tilde{A}_{2}\right)$ then $\tilde{A}_{1}=\tilde{A}_{2}$

\section{III.ARITHMETIC OPERATIONS BETWEEN TWO SV-}

TRAPEZOIDAL NEUTROSOPHIC NUMBERS

In this subsection, we make a slight modification of the operations between single valued trapezoidal neutrosophic numbers proposed by Deli and Subas [11], required for the proposed algorithm.

Let $\tilde{A}_{1}=<\left(\mathrm{a}_{1}, a_{2}, a_{3}, a_{4}\right) ; T_{1}, \mathrm{I}_{1}, \mathrm{~F}_{1}>$ and $\tilde{A}_{2}=<\left(b_{1}, b_{2}, b_{3}, b_{4}\right) ; T_{2}, \mathrm{I}_{2}, \mathrm{~F}_{2}>$ be two single valued trapezoidal neutrosophic number. Then, the operations for SVTNNs are defined as below;

(i)

$$
\tilde{A}_{1} \oplus \tilde{A}_{2}=<\left(\mathrm{a}_{1}+b_{1}, \mathrm{a}_{2}+b_{2}, \mathrm{a}_{3}+b_{3}, \mathrm{a}_{4}+b_{4}\right) ; T_{1}+T_{2}-T_{1} T_{2}, \mathrm{I}_{1} \mathrm{I}_{2}, \mathrm{~F}_{1} \mathrm{~F}_{2}>
$$

(ii)

$$
\begin{aligned}
& \left.\tilde{A}_{1} \otimes \tilde{A}_{2}=<\left(\mathrm{a}_{1} b_{1}, \mathrm{a}_{2} b_{2}, \mathrm{a}_{3} b_{3}, \mathrm{a}_{4} b_{4}\right) ; T_{1} T_{2}, \mathrm{I}_{1}+\mathrm{I}_{2}-\mathrm{I}_{1} \mathrm{I}_{2}, \mathrm{~F}_{1}+\mathrm{F}_{2}-\mathrm{F}_{1} \mathrm{~F}_{2}\right)> \\
& \text { (iii) } \left.\left.\lambda \tilde{A}_{1}=<\left(\lambda \mathrm{a}_{1}, \lambda \mathrm{a}_{2}, \lambda \mathrm{a}_{3}, \lambda \mathrm{a}_{4}\right) ; 1-\left(1-T_{1}\right)^{\lambda}\right), \mathrm{I}_{1}^{\lambda}, F_{1}^{\lambda}\right)>
\end{aligned}
$$

\section{NETWORK TERMINOLOGY}

Consider a directed network $\mathrm{G}=(\mathrm{V}, \mathrm{E})$ consisting of a finite set of nodes $V=\{1,2, \ldots, n\}$ and a set of $m$ directed edges $\mathrm{E} \subseteq \mathrm{V} \times \mathrm{V}$. Each edge is denoted by an ordered pair $(i, j)$ where $\mathrm{i}, \mathrm{j} \in \mathrm{V}$ and $i \neq j$. In this network, we specify two nodes, denoted by $s$ and $t$, which are the source node and the destination node, respectively. We define a path as a sequence $P_{i j}=\left\{\mathrm{i}=i_{1},\left(i_{1}, i_{2}\right), i_{2}, \ldots, i_{l-1},\left(i_{l-1}, i_{l}\right), i_{l}=\mathrm{j}\right\}$ of alternating nodes and edges. The existence of at least one path $P_{s i}$ in $\mathrm{G}$ $(\mathrm{V}, \mathrm{E})$ is assumed for every $\mathrm{i} \in \mathrm{V}-\{\mathrm{s}\}$.

$d_{i j}$ denotes a single valued trapezoidal neutrosophic number associated with the edge $(i, j)$, corresponding to the length necessary to traverse $(i, j)$ from $i$ to $j$. In real problems, the lengths correspond to the cost, the time, the distance, etc. Then, neutrosophic distance along the path $\mathrm{P}$ is denoted as $\mathrm{d}(\mathrm{P})$ is defined as

$$
\mathrm{d}(\mathrm{P})=\sum_{(\mathrm{i}, \mathrm{j} \in \mathrm{P})} d_{i j}
$$

Remark : A node $i$ is said to be predecessor node of node $j$ if (i) Node $\mathrm{i}$ is directly connected to node $\mathrm{j}$.

(ii) The direction of path connecting node $i$ and $j$ from $i$ to $j$.

\section{V . SINGLE VALUED TRAPEZOIDAL NEUTROSOPHIC} PATH PROBLEM

In this section, motivated by the work of Kumar [14], an algorithm is proposed to find the path of minimum distance between the source node (i) and the destination node (j) in a single valued trapezoidal neutrosophic graph.

The main steps of the algorithm are as follows:

Step 1 Assume $\tilde{d}_{1}=<(0,0,0,0) ; 0,1,1>$ and label the source node (say node1) as $\left[\tilde{d}_{1}=<(0,0,0,0) ; 0,1,1>,-\right]$. The label indicating that the node has no predecessor.

Step 2 Find $\tilde{d}_{j}=\operatorname{minimum}\left\{\tilde{d}_{i} \oplus \tilde{d}_{i j}\right\} ; \mathrm{j}=2,3, \ldots, \mathrm{n}$.

Step 3 If minimum occurs corresponding to unique value of $i$ i.e., $\mathrm{i}=\mathrm{r}$ then label node $\mathrm{j}$ as $\left[\tilde{d}_{j}, \mathrm{r}\right]$. If minimum occurs corresponding to more than one values of $i$ then it represents that there are more than one single valued trapezoidal neutrosophic path between source node and node $\mathrm{j}$ but single valued trapezoidal neutrosophic distance along path is $\tilde{d}_{j}$, so choose any value of $i$.

Step 4 Let the destination node (node $\mathrm{n}$ ) be labeled as $\left[\tilde{d}_{n}, l\right]$, then the single valued trapezoidal neutrosophic shortest distance between source node and destination node is $\tilde{d}_{n}$.

Step 5 Since destination node is labeled as $\left[\tilde{d}_{n}, l\right]$, so, to find the single valued trapezoidal neutrosophic shortest path between source node and destination node, check the label of node $l$. Let it be $\left[\tilde{d}_{l}, \mathrm{p}\right]$, now check the label of node $\mathrm{p}$ and so on. Repeat the same procedure until node 1 is obtained.

Step 6 Now the single valued trapezoidal neutrosophic shortest path can be obtained by combining all the nodes obtained by the step 5 .

Remark 5.1 Let $\tilde{A}_{i} ; \mathrm{i}=1,2, \ldots, \mathrm{n}$ be a set of single valued trapezoidal neutrosophic numbers, if $\mathrm{S}\left(\tilde{A}_{k}\right)<\mathrm{S}\left(\tilde{A}_{i}\right)$, for all i, the single valued trapezoidal neutrosophic number is the minimum of $\tilde{A}_{k}$.

After describing the proposed algorithm, in next section we solve a numerical example and explain the proposed method completely.

\section{ILLUSTRATIVE EXAMPLE}

Now we solve an hypothetical example to verify the proposed approach. Consider the network shown in figure1, we want to obtain the shortest path from node 1 to node 6 where edges have a single valued trapezoidal neutrosophic numbers. Let us now apply the proposed algorithm to the network given in figure 1 .

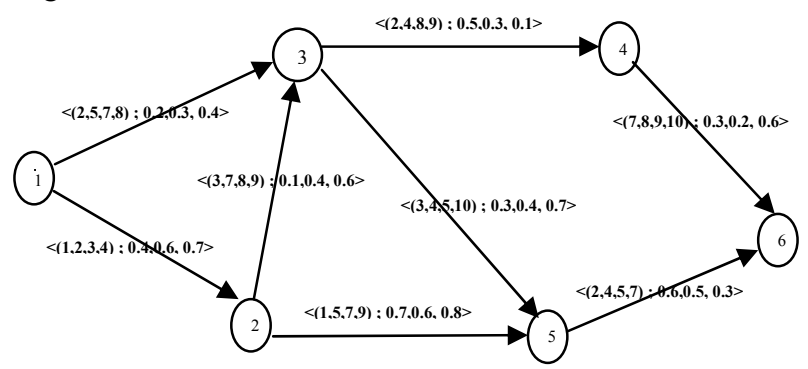


Fig. 1. A network with single valued trapezoidal neutrosophic edges

In this network each edge have been assigned to single valued trapezoidal neutrosophic number as follows:

\begin{tabular}{|l|l|}
\hline Edges & $\begin{array}{c}\text { single valued trapezoidal } \\
\text { Neutrosophic distance }\end{array}$ \\
\hline $1-2$ & $<(1,2,3,4) ; 0.4,0.6,0.7>$ \\
\hline $1-3$ & $<(2,5,7,8) ; 0.2,0.3,0.4>$ \\
\hline $2-3$ & $<(3,7,8,9) ; 0.1,0.4,0.6>$ \\
\hline $2-5$ & $<(1,5,7,9) ; 0.7,0.6,0.8>$ \\
\hline $3-4$ & $<(2,4,8,9) ; 0.5,0.3,0.1>$ \\
\hline $3-5$ & $<(3,4,5,10) ; 0.3,0.4,0.7>$ \\
\hline $4-6$ & $<(7,8,9,10) ; 0.3,0.2,0.6>$ \\
\hline $5-6$ & $<(2,4,5,7) ; 0.6,0.5,0.3>$ \\
\hline
\end{tabular}

Table 1. Weights of the graphs

The calculations for this problem are as follows: since node 6 is the destination node, so $n=6$.

Assume $\tilde{d}_{1}=<(0,0,0,0) ; 0,1,1>$ and label the source node ( say node 1$)$ as $[<(0,0,0,0) ; 0,1,1\rangle,-]$, the value of $\tilde{d}_{j} ; \mathrm{j}=2,3,4,5,6$ can be obtained as follows:

Iteration 1 Since only node 1 is the predecessor node of node 2 , so putting $\mathrm{i}=1$ and $\mathrm{j}=2$ in step 2 of the proposed algorithm, the value of $\tilde{d}_{2}$ is

$\tilde{d}_{2}=\operatorname{minimum}\left\{\tilde{d}_{1} \oplus \tilde{d}_{12}\right\}=\operatorname{minimum}\{<(0,0,0,0) ; 0,1,1>\oplus$ $<(1,2,3,4) ; 0.4,0.6,0.7>=<(1,2,3,4) ; 0.4,0.6,0.7>$

Since minimum occurs corresponding to $\mathrm{i}=1$, so label node 2 as $[<(1,2,3,4) ; 0.4,0.6,0.7>, 1]$

Iteration 2 The predecessor node of node 3 are node 1 and node 2 , so putting $i=1,2$ and $j=3$ in step 2 of the proposed algorithm, the value of $\tilde{d}_{3}$ is

$\tilde{d}_{3}=\operatorname{minimum}\left\{\tilde{d}_{1} \oplus \tilde{d}_{13}, \tilde{d}_{2} \oplus \tilde{d}_{23}\right\}=\operatorname{minimum}\{<(0,0,0,0) ; 0$, $1,1>\oplus<(2,5,7,8) ; 0.2,0.3,0.4>,<(1,2,3,4) ; 0.4,0.6$, $0.7>\oplus<(3,7,8,9) ; 0.1,0.4,0.6>\}=\operatorname{minimum}\{<(2,5,7,8)$; $0.2,0.3,0.4>,<(4,9,11,13) ; 0.46,0.24,0.42>\}$

$\mathrm{S}(\{<(2,5,7,8) ; 0.2,0.3,0.4>)$

$=\left(\frac{1}{12}\right)\left[a_{1}+a_{2}+a_{3}+a_{4}\right] \times\left[2+T_{1}-I_{1}-F_{1}\right]=2.75$

$=$

$\mathrm{S}(<(4,9,11,13) ; 0.46,0.24,0.42>)=5.55$

Since $\mathrm{S}(\{<(2,5,7,8) ; 0.2,0.3,0.4>)<\mathrm{S}(<(4,9,11,13)$; $0.46,0.24,0.42>)$
So minimum $\{<(2,5,7,8) ; 0.2,0.3,0.4>,<(4,9,11,13)$; $0.46,0.24,0.42>\}$

$=<(2,5,7,8) ; 0.2,0.3,0.4>$

Since minimum occurs corresponding to $\mathrm{i}=1$, so label node 3 as $[<(2,5,7,8) ; 0.2,0.3,0.4>, 1]$

Iteration 3. The predecessor node of node 4 is node 3, so putting $\mathrm{i}=3$ and $\mathrm{j}=4$ in step 2 of the proposed algorithm, the value of $\tilde{d}_{4}$ is $\tilde{d}_{4}=\operatorname{minimum}\left\{\tilde{d}_{3} \oplus \tilde{d}_{34}\right\}=\operatorname{minimum}\{<(2,5$, $7,8) ; 0.2,0.3,0.4>, \oplus<(2,4,8,9) ; 0.5,0.3,0.1>\}=<(4,9$, $15,17) ; 0.6,0.09,0.04>$

So minimum $\{<(2,5,7,8) ; 0.2,0.3,0.4>, \oplus<(2,4,8,9)$; $0.5,0.3,0.1>\}=<(4,9,15,17) ; 0.6,0.09,0.04>$

Since minimum occurs corresponding to $\mathrm{i}=3$, so label node 4 as $[<(4,9,15,17) ; 0.6,0.09,0.04>, 3]$

Iteration 4 The predecessor node of node 5 are node 2 and node 3 , so putting $i=2,3$ and $j=5$ in step 2 of the proposed algorithm, the value of $\tilde{d}_{5}$ is

$\tilde{d}_{5}=\operatorname{minimum}\left\{\tilde{d}_{2} \oplus \tilde{d}_{25}, \tilde{d}_{3} \oplus \tilde{d}_{35}\right\}=\operatorname{minimum}\{<(1,2,3,4)$;

$0.4,0.6,0.7>\oplus<(1,5,7,9) ; 0.7,0.6,0.8>,<(2,5,7,8) ; 0.2$, $0.3,0.4>\oplus<(3,4,5,10) ; 0.3,0.4,0.7>\}=$

minimum $\{<(2,7,10,13) ; 0.82,0.36,0.56>,<(5,9,12,18)$; $0.44,0.12,0.28>\}$

$\mathrm{S}(<(2,7,10,13) ; 0.82,0.36,0.56>)=5.06$

$\mathrm{S}(<(5,9,12,18) ; 0.44,0.12,0.28>)=7.48$

Since $\mathrm{S}(<(2,7,10,13) ; 0.82,0.36,0.56>)<\mathrm{S}(<(5,9,12$, $18) ; 0.44,0.12,0.28>)$

minimum $\{<(2,7,10,13) ; 0.82,0.36,0.56>,<(5,9,12,18)$; $0.44,0.12,0.28>\}$

$=<(2,7,10,13) ; 0.82,0.36,0.56>$

$\tilde{d}_{5}=<(2,7,10,13) ; 0.82,0.36,0.56>$

Since minimum occurs corresponding to $i=2$, so label node 5 as $[<(2,7,10,13) ; 0.82,0.36,0.56>, 2]$

Iteration 5 The predecessor node of node 6 are node 4 and node 5 , so putting $i=4$, 5and $j=6$ in step 2 of the proposed algorithm, the value of $\tilde{d}_{6}$ is

$\tilde{d}_{6}=\operatorname{minimum}\left\{\tilde{d}_{4} \oplus \tilde{d}_{46}, \tilde{d}_{5} \oplus \tilde{d}_{56}\right\}=\operatorname{minimum}\{<(4,9,15,17)$; $0.6,0.09,0.04>\oplus<(7,8,9,10) ; 0.3,0.2,0.6>,<(2,7,10$, 13); $0.82,0.36,0.56>\oplus<(2,4,5,7) ; 0.6,0.5,0.3>\}=$ minimum $\{<(11,17,24,27) ; 0.72,0.018,0.024>,<(4,11,15$, $20) ; 0.93,0.18,0.17>$ \}

$\mathrm{S}(<(11,17,24,27) ; 0.72,0.018,0.024>)=17.63$

$\mathrm{S}(<(4,11,15,20) ; 0.93,0.18,0.17>)=10.75$

Since S $(<(4,11,15,20) ; 0.93,0.18,0.17>)<\mathrm{S}(<(11,17$, $24,27) ; 0.72,0.018,0.024>)$

So minimum $\{<(11,17,24,27) ; 0.72,0.018,0.024>,<(4,11$, $15,20) ; 0.93,0.18,0.17>\}$

$=<(4,11,15,20) ; 0.93,0.18,0.17>$

$\tilde{d}_{6}=<(4,11,15,20) ; 0.93,0.18,0.17>$

Since minimum occurs corresponding to $i=5$, so label node 6 as $[<(4,11,15,20) ; 0.93,0.18,0.17>, 5]$

Since node 6 is the destination node of the given network, so the single valued trapezoidal neutrosophic shortest distance 
between node 1 and node 6 is $<(4,11,15,20)$; $0.93,0.18$, $0.17>$.

Now the single valued trapezoidal neutrosophic shortest path between node 1 and node 6 can be obtained by using the following procedure:

Since node 6 is labeled by $[<(4,11,15,20) ; 0.93,0.18,0.17>$, 5], which represents that we are coming from node 5 . Node 5 is labeled by $[<(2,7,10,13) ; 0.82,0.36,0.56>, 2]$, which represents that we are coming from node 2 . Node 2 is labeled by $[<(1,2,3,4) ; 0.4,0.6,0.7>, 1]$, which represents that we are coming from node 1 . Now the single valued trapezoidal neutrosophic shortest path between node 1 and node 6 is obtaining by joining all the obtained nodes. Hence the single valued trapezoidal neutrosophic shortest path is $1 \rightarrow 2 \rightarrow 5 \rightarrow 6$

The single valued trapezoidal neutrosophic shortest distance and the single valued trapezoidal neutrosophic shortest path of all nodes from node 1 is shown in the table 2 and the labeling of each node is shown in figure 2

\begin{tabular}{|l|l|l|}
\hline $\begin{array}{l}\text { Node } \\
\text { No.(j) }\end{array}$ & $\tilde{d}_{i}$ & $\begin{array}{c}\text { Single valued } \\
\text { trapezoidal } \\
\text { Neutrosophic shortest } \\
\text { path between jth and } \\
\text { 1st node }\end{array}$ \\
\hline 2 & $<(1,2,3,4) ; 0.4,0.6,0.7>$ & $1 \rightarrow 2$ \\
\hline 3 & $<(2,5,7,8) ; 0.2,0.3,0.4>$ & $1 \rightarrow 3$ \\
\hline 4 & $<(4,9,15,17) ; 0.6,0.09,0.04>$ & $1 \rightarrow 3 \rightarrow 4$ \\
\hline $\mathbf{5}$ & $<(2,7,10,13) ; 0.82,0.36,0.56>$ & $1 \rightarrow 2 \rightarrow 5$ \\
\hline 6 & $<(4,11,15,20) ; 0.93,0.18,0.17>$ & $1 \rightarrow 2 \rightarrow 5 \rightarrow 6$ \\
\hline
\end{tabular}

Table 2. Tabular representation of different single valued trapezoidal neutrosophic shortest paths

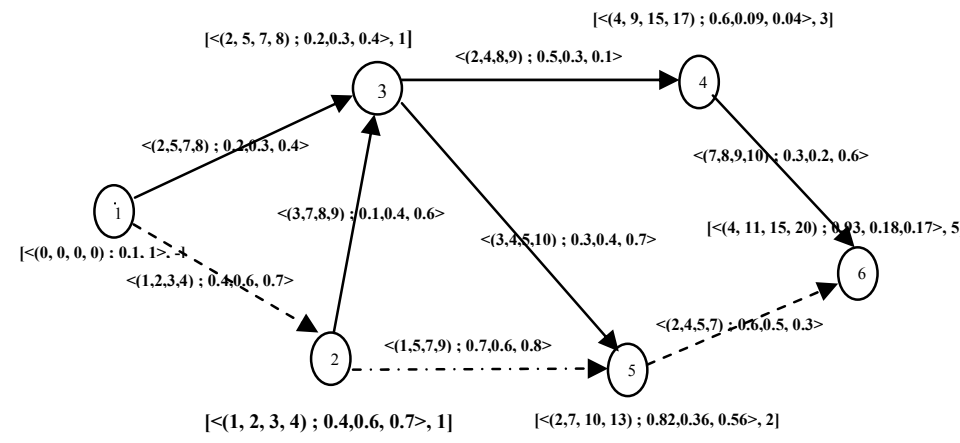

FIG 2. Network with single valued trapezoidal neutrosophic shortest distance of each node from node 1

\section{CONCLUSION}

In this paper, we have developed an algorithm for solving shortest path problem on a network with single valued trapezoidal neutrosophic edge lengths. The process of ranking the path is very useful to make decisions in choosing the best of all possible path alternatives. Numerical example via six node network showed the performance of the proposed methodology for the shortest path. Next, we will research the application of this algorithm.

\section{Acknowledgment}

The authors are very grateful to the chief editor and reviewers for their comments and suggestions, which is helpful in improving the paper.

\section{REFERENCES}

[1] F. Smarandache, "Neutrosophic set - a generalization of the intuitionistic fuzzy set," Granular Computing, 2006 IEEE International Conference, 2006 , p. $38-42$.

[2] F. Smarandache, "A geometric interpretation of the neutrosophic set - A generalization of the intuitionistic fuzzy set," Granular Computing (GrC), 2011 IEEE International Conference, 2011, pp.602606 .

[3] L. Zadeh, Fuzzy sets. Inform and Control, 8, 1965, pp.338-353

[4] K. Atanassov, "Intuitionistic fuzzy sets," Fuzzy Sets and Systems, vol. 20, 1986, pp. 87-96.

[5] I. Turksen, "Interval valued fuzzy sets based on normal forms," Fuzzy Sets and Systems, vol. 20,1986, pp. 191-210.

[6] K. Atanassov and G. Gargov, Interval valued intuitionistic fuzzy sets. Fuzzy Sets and Systems, vol.31, 1989, pp.343-349.

[7] H. Wang, F. Smarandache, Y. Zhang, and R. Sunderraman, "Single valued Neutrosophic Sets," Multisspace and Multistructure 4, 2010, pp. 410-413.

[8] http://fs.gallup.unm.edu/NSS

[9] Y. Subas, Neutrosophic numbers and their application to multiattribute decision making problems,( in Turkish) ( master Thesis, 7 Aralk university, Graduate School of Natural and Applied Science, 2015.

[10] P. Biswas, S. Parmanik and B. C. Giri, Cosine Similarity Measure Based Multi-attribute Decision-Making with Trapezoidal fuzzy Neutrosophic numbers, Neutrosophic sets and systems, 8, 2014,pp. 47-57.

[11] I. Deli and Y. Subas, A Ranking methods of single valued neutrosophic numbers and its application to multi-attribute decision making problems, International Journal of Machine Learning and Cybernetics, 2016,pp.1-14.

[12] A. Thamaraiselvi and R.Santhi, A New Approach for Optimization of Real Life Transportation Problems in Neutrosophic Environment, Mathematical Problems in Enginnering,2016, 9 pages.

[13] P. Jayagowri and G. Geetha Ramani, Using Trapezoidal Intuitionistic Fuzzy Number to Find Optimized Path in a Network, Volume 2014, Advances in Fuzzy Systems, 2014, 6 pages

[14] A. Kumar and M. Kaur, A New Algorithm for Solving Shortest Path Problem on a Network with Imprecise Edge Weight, Applications and Applied Mathematics, Vol. 6, Issue 2, 2011, pp. $602-619$.

5 ] A. Kumar, and M. Kaur, Solution of fuzzy maximal flow oblems using fuzzy linear programming. World Academy of Science and Technology. 87, 2011,pp. 28-31.

[16] S. Majumder and A. Pal, Shortest Path Problem on Intuitionistic Fuzzy Network, Annals of Pure and Applied Mathematics, Vol. 5, No. 1, November 2013, pp. 26-36.

[17] S. Broumi, M. Talea, A. Bakali, F. Smarandache, "Single Valued Neutrosophic Graphs," Journal of New Theory, N 10, 2016, pp. 86-101.

[18] S. Broumi, M. Talea, A. Bakali, F. Smarandache, "On Bipolar Single Valued Neutrosophic Graphs," Journal of New Theory, N11, 2016, pp.84-102.

[19] S. Broumi, M. Talea, A. Bakali, F. Smarandache, Interval Valued Neutrosophic Graphs, (2016) in press.

[20] S. Broumi, A. Bakali, M, Talea, and F, Smarandache, Isolated Single Valued Neutrosophic Graphs. Neutrosophic Sets and Systems, Vol. 11, 2016, pp.74-78

[21] S. Broumi, F. Smarandache, M. Talea and A. Bakali, An Introduction to Bipolar Single Valued Neutrosophic Graph Theory. Applied Mechanics and Materials, vol.841, 2016, pp. 184-191.

[22] S. Broumi, M. Talea, F. Smarandache and A. Bakali, Single Valued Neutrosophic Graphs: Degree, Order and Size, 2016 IEEE International Conference on Fuzzy Systems (FUZZ), 2016, pp.2445-2451

[23] S. Broumi, A. Bakali, M. Talea and F. Smarandache, Computation of Shortest Path Problem in a Network with Single Valued Neutrosophic 
Number Based on Ranking Method, 2016 (submitted)

[24] S. Broumi, A. Bakali, M. Talea, F. Smarandache, M. Ali, Shortest Path Problem Under Bipolar Neutrosophic Setting, 2016 (submitted).

[25] S. Broumi, A. Bakali, M. Talea and F. Smarandache, Shortest Path Problem Under Interval Valued Neutrosophic Setting, 2016 (submitted). 